\title{
Cosa ha da dirci Paulo Freire a cento anni dalla sua nascita
}

\section{O que Paulo Freire tem a nos dizer há cem anos do seu nascimento}

\section{What Paulo Freire has to tell us one hundred years after his birth}

\author{
Massimiliano Fiorucci*
}

Pensare la storia come possibilità significa riconoscere l'educazione come possibilità. Significa riconoscere che, anche se l'educazione non può fare tutto da sola, può però certo raggiungere qualche risultato. La sua forza sta nella sua debolezza. Paulo Freire (1971).

\section{RIASSUNTO}

Il contributo si sofferma sulla figura di Paulo Freire a cento anni dalla sua nascita con l'obiettivo di sottolineare come sia importante ancora oggi leggere questo autore per comprendere e decodificare il presente e far emergere le forme di oppressione ancora presenti nel mondo contemporaneo. Il contributo, inoltre, evidenzia il rapporto tra educazione e politica in Freire e il suo impegno profetico sui temi dell'educazione interculturale.

Parole Chiave: Paulo Freire. Educazione interculturale. Italia.

\section{RESUMO}

A contribuição centra-se na figura de Paulo Freire cem anos após o seu nascimento, com o objetivo de enfatizar a importância da leitura deste autor para compreender e decodificar o presente e trazer à tona as formas de

*Università degli Studi Roma Tre. Roma, Itália. E-mail: massimiliano.fiorucci@uniroma3. it - https://orcid.org/ 0000-0002-9927-6095 
opressão ainda presentes no mundo contemporâneo. A contribuição também destaca a relação entre educação e política em Freire e seu compromisso profético com as questões da educação intercultural.

Palavras-Chave: Paulo Freire. Educação intercultural. Itália.

\begin{abstract}
The present paper focuses on the figure of Paulo Freire one hundred years after his birth. It aims at emphasizing how nowadays it is important to read Freire to understand and decode the present and to bring to light the forms of oppression that are still present in contemporary world. This contribution also highlights the relationship between education and politics in Freire's philosophy and his prophetic commitment related to issues of intercultural education.
\end{abstract}

Keywords: Paulo Freire. Intercultural education. Italy.

Cento anni fa, il 19 settembre 1921, nasceva in Brasile Paulo Freire, destinato a diventare una pietra miliare della pedagogia mondiale, con un una visione e una prospettiva, costate anche l'esilio, che hanno fortemente influenzato il modo di intendere l'educazione in chiave emancipativa. In una inedita elaborazione intellettuale, ben nutrita dallo studio del personalismo, del marxismo, della teologia della liberazione, da un orizzonte culturale intimamente cristiano e sensibile alle istanze anticoloniali, Freire si è posto fin dagli anni '60 come un grande innovatore del pensiero pedagogico, con una teoria e una visione della prassi che ancora oggi, con le contraddizioni del mondo contemporaneo, sembrano parlare tanto al tempo presente quanto a quello futuro.

Il mondo in cui opera Paulo Freire è quello della guerra fredda e della decolonizzazione e, più nello specifico, quella realtà così densa di ingiustizie che è il Brasile dell'epoca. Nato a Recife, dopo una formazione giuridica, ma anche filosofica e psicologica, nel 1946 viene nominato Direttore del Dipartimento di Educazione e Cultura del Servizio Sociale nello Stato del Pernambuco. Già da questo momento, in un contesto in cui il diritto al voto è garantito a chi possiede un'istruzione, Freire inizia a mostrare forte attenzione agli adulti analfabeti, in una cornice teorica, non distante dalla teologia della liberazione, in cui confluiscono la sua fede cristiana e una visione vicina al marxismo gramsciano. Nei primissimi anni '60 l'applicazione del suo metodo per consentire a soggetti adulti di apprendere a leggere e a scrivere, stimola la nascita di migliaia di circoli culturali dediti alla lotta all'analfabetismo. Arrivano, con la dittatura militare, 
gli anni dell'esilio durante i quali si è impegnato per il Movimento Cristiano Democratico di Riforma Agraria in Cile. È in questo periodo che Freire inizia a scrivere le sue più importanti opere, destinate a diventare un riferimento imprescindibile per la pedagogia mondiale e per quei mondi dell'educazione in tensione con le grandi ingiustizie e le disuguaglianze: "L'educazione come pratica della libertà" (1967), "La pedagogia degli oppressi" (1968), "Pedagogia della speranza. Un nuovo approccio alla pedagogia degli oppressi" (1992).

Dal 1980, tornato in Brasile, si dedica all'attività politica con il Partido dos Trabalhadores, ricopre incarichi di responsabilità in campo educativo e continua la sua meritoria opera in campo pedagogico. Morirà nel 1997. Inutile sottolineare quanto la sua pedagogia degli oppressi sia stata rilevante per ispirare il grande regista brasiliano Augusto Boal nell'affinare le prospettive e le tecniche del noto Teatro dell'oppresso.

A cento anni dalla nascita di Paulo Freire si può considerare ancora attuale la sua pedagogia? Il suo metodo educativo può contribuire alla chiarificazione dei problemi educativi nelle odierne società consumistiche e globalizzate? Perché continuare a leggere Freire? Era esattamente questo il titolo della prefazione di Moacir Gadotti alla nuova edizione italiana de "La pedagogia degli oppressi" apparsa nel 2002 a distanza di più di trent'anni dalla prima edizione del 1971 curata e introdotta da Laura Bimbi (FREIRE, 1971). Oggi la domanda di Moacir Gadotti rimane ancora valida e di stringente attualità: è necessario leggere e/o rileggere Freire perché sono ancora tante le forme di oppressione (più o meno evidenti) presenti nelle nostre società e Freire ci ricorda che l'educazione, la cultura, la scienza sono prima di tutto uno strumento di liberazione e di emancipazione.

Non è possibile in questa sede individuare tutte le forme di oppressione presenti nella nostra società ma sicuramente possono essere individuate alcune categorie di soggetti oppressi: coloro che sono in possesso di deboli livelli di istruzione e che non sono in grado di effettuare scelte del tutto libere e consapevoli, i migranti che, nella maggior parte dei casi, si vedono costretti ad accettare e a svolgere i cosiddetti lavori delle 5P (precari, pesanti, poco pagati, penalizzati socialmente, pericolosi) in un quadro generale di integrazione "subalterna" o di inclusione subordinata, le donne che a parità di titoli di studio guadagnano meno degli uomini e solo raramente riescono a raggiungere posizioni apicali, i NEET (Not in Education, Employment or Training), una parte dei giovani, i precari e così via.

Qualche dato sull'istruzione in Italia può aiutare a meglio definire la questione. I dati drammatici sulla dispersione scolastica e sui cosiddetti NEETs 
sono molto eloquenti in proposito ${ }^{1}$. Una situazione così grave non caratterizza solamente la scuola dell'obbligo. Se si considera il numero di quanti sono in possesso di un titolo di studio universitario, l'Italia si colloca in fondo alle classifiche europee. Un ulteriore elemento critico riguarda la popolazione adulta e quello che viene definito "analfabetismo funzionale": i cittadini italiani si collocano in fondo alla classifica sui saperi essenziali per orientarsi nella società del terzo millennio. L'identità reale del sistema sociale e formativo come quello italiano - che ancora opera una distribuzione differenziata delle conoscenze sulla base di fattori di ordine sociale, di genere, territoriale e di nazionalità contraddice l'autorappresentazione che la nostra società ha di sé stessa come di una società moderna che a tutti fornirebbe le stesse opportunità di vita e di lavoro. Si tratta in altri termini di una società ancora fortemente divisa, per usare una terminologia introdotta da Paulo Freire, in oppressori e oppressi. Ciò determina una situazione assai pericolosa in cui vi sono alcune persone (poche) in grado di operare scelte libere e consapevoli esercitando il pensiero critico e tante altre (troppe) che non dispongono degli strumenti minimi per decostruire le false rappresentazioni e le facili semplificazioni delle demagogie e dei populismi.

Cosa può dirci allora oggi Paulo Freire?

L'opera di Paulo Freire si caratterizza come una visione pedagogica complessiva: una sorta di pedagogia dell'uomo e del dialogo densa di aspetti e di riferimenti antropologici, sociologici e filosofici. Freire, infatti, fu un alfabetizzatore ed educatore degli adulti non solo in Brasile, ma anche in Cile e nell'Africa delle ex colonie portoghesi, mentre allo stesso tempo teneva contatti con Università e altre istituzioni educative nordamericane, svizzere e anche italiane. Anche in conseguenza di questi elementi vi è chi ne ha parlato come di un vero e proprio "educatore del mondo" (TAGLIAVIA, 2011). Tra i tanti riferimenti del pensiero freireano vi sono il personalismo cristiano del filosofo francese Jacques Maritain (1882-1973) - un'influenza che appartiene soprattutto alla prima fase della sua elaborazione; le teorie sul linguaggio dello psicologo culturale sovietico Lev Semenovic Vygotskij (1896-1934) - con particolare riferimento alla parte del lavoro specificamente dedicata al Metodo

1 Un recentissimo Dossier di "Tuttoscuola" (La scuola colabrodo, settembre 2018), confrontando il numero di quanti sono entrati in istituti tecnici, professionali o licei e quanti ne sono usciti cinque anni dopo con un titolo, dal 1995 a oggi, mostra come l'Italia abbia perso lungo la strada tre milioni e mezzo di studenti dal 1995 a oggi. L'Italia ha anche il primato dei cosiddetti NEETs: secondo Eurostat l'Italia si conferma maglia nera in Europa per la quota di giovani tra i 18 e 24 anni che non hanno un lavoro né sono all'interno di un percorso di studi o di formazione. Il nostro Paese primeggia nel 2017 nella classifica europea, con una percentuale del $25,7 \%$, a fronte di una media europea del $14,3 \%$. 
Paulo Freire; la teoria dell'egemonia e della subalternità del filosofo e politico italiano Antonio Gramsci (1891-1937), che peraltro avvicina il pedagogista brasiliano alla corrente degli studi post-coloniali.

Paulo Freire, nel quadro delle pedagogie del Novecento, rappresenta senza dubbio uno degli autori che possono essere definiti come pensatori critici. L'importante pedagogista brasiliano, infatti, durante tutta la sua attività scientifica e militante individua due concezioni dell'educazione tra loro contrapposte: l'educazione "depositaria" (termine con cui viene tradotto il portoghese bancària) e l'educazione "problematizzante". L'una conserva e conferma, l'altra produce consapevolezza critica ed è il presupposto della liberazione. L'educazione, infatti, diventa per Paulo Freire un percorso di liberazione.

Freire scrive la sua opera principale, "La pedagogia degli oppressi", nel 1968. Egli è un testimone significativo dell'America Latina degli anni '60: un contesto oggetto di molti mutamenti politici e di svolte autoritarie che a volte portarono, come nel caso del Brasile nel 1964, a violente dittature militari. Freire, dopo aver partecipato come educatore all' Istituto Superiore di Educazione Brasiliana (ISEB) e a varie iniziative di alfabetizzazione delle popolazioni rurali, fu costretto all'esilio: visse dapprima in Cile, dove pubblicò "La pedagogia degli oppressi" e altre opere fondamentali della sua produzione - tra cui "L'educazione come pratica della libertà" - e poi in Svizzera.

L'educazione depositaria, secondo Freire, è un modello di educazione direttiva e ingiusta, in cui l'educatore educa e gli educandi sono educati, l'educatore sa e gli educandi non sanno, l'educatore parla e gli educandi ascoltano docilmente. L'educazione problematizzante, al contrario,

[...] è intenzionalità, perché risposta a ciò che la coscienza profondamente è, e quindi rifiuta i comunicati e rende essenzialmente vera la comunicazione... In questo senso, l'educazione liberatrice, problematizzante, non può essere l'atto di depositare, o di narrare, o di trasferire, o di trasmettere conoscenze e valori agli educandi, semplici, pazienti, come succede nell'educazione depositaria, bensì un atto di conoscenza" (FREIRE, 2002, p. 67-68).

Questa concezione dialogica dell'educazione e dell'atto di insegnare, fondamentale nel pensiero freiriano, verrà ripresa anche in uno degli ultimi scritti del pedagogista brasiliano, "Pedagogia dell'autonomia", interamente dedicato al tema della formazione docente, in cui egli afferma che "chi insegna, nell'atto di insegnare apprende, e chi apprende nell'atto di farlo, insegna" (FREIRE, 2004, p. 21). E ancora: 
[...] insegnare, apprendere e ricercare hanno a che fare con questi due momenti del ciclo gnoseologico: quello in cui si insegna e si apprende la conoscenza già esistente, e quello in cui si lavora all'elaborazione della conoscenza che ancora non esiste. La «do-discenza» - la docenza-discenza - e la ricerca finiscono così con l'essere pratiche essenziali - e inseparabili - di questi momenti del ciclo gnoseologico (FREIRE, 2004, p. 25).

Sempre in questo volume, pubblicato per la prima volta nel 1966, si ribadisce che

[...] uno dei saperi indispensabili è che chi viene formato, fin dall'inizio della sua esperienza, si consideri egli stesso un soggetto che produce sapere, e si convinca una volta per tutte che insegnare non è trasferire conoscenza, ma creare le possibilità per produrla o costruirla (FREIRE, 2004, p. 24).

Formare, in altri termini, è molto più che addestrare una persona, grande o piccola, giovane o adulta, nell'uso di alcune abilità. Insegnare esige comprendere che educare è una forma di intervento sul mondo; l'educazione non è mai stata, non è, né può essere neutrale, “indifferente”. Freire sottolinea come non vi sia insegnamento senza apprendimento e, pertanto, insegnare esige ricerca, capacità critica e riflessione critica sulla pratica. Insegnare, come si è detto, non è trasferire conoscenza ed esige curiosità passando da una curiosità ingenua ad una curiosità epistemologica. Insegnare, come sottolinea Freire, è una peculiarità umana ed esige che ci si impegni comprendendo che educare è una forma di intervento sul mondo. Gli insegnanti e gli educatori devono saper ascoltare ed essere disponibili al dialogo riconoscendo che l'educazione è ideologica prendendo posizione sulle questioni importanti.

Le tematiche principali affrontate da Freire nel volume "La pedagogia degli oppressi" riguardano l'interpretazione della realtà come dinamica di oppressione/liberazione, la concezione problematizzante dell'educazione, il concetto di dialogo e di anti-dialogo, gli aspetti metodologici del processo di alfabetizzazione. Quest'ultimo viene ritenuto centrale non solo come metodo "scientifico" per imparare a leggere e a scrivere, ma anche come condizione prioritaria per la partecipazione politica, e la conseguente liberazione, dell'oppresso. La dialettica oppresso/oppressore richiama, come evidenzia esplicitamente Freire stesso, la dialettica hegeliana servo/signore: l'oppresso, 
per Freire come per Hegel, acquistando la propria umanità con il processo di liberazione, restituisce umanità anche al proprio oppressore, liberandolo. Le due fonti principali del pensiero di Freire sono dunque il personalismo cristiano di J. Maritain e il marxismo (la realtà come dialettica oppressori/oppressi e l'educazione come strumento di trasformazione della realtà).

Per quanto riguarda, in particolare, il metodo Paulo Freire, esso si basa sulle "parole generatrici" e sui "quadri-situazione". Queste due strategie permettono di focalizzare l'attenzione su un tema che permette dapprima un processo di "coscientizzazione", da parte dell'analfabeta, della sua condizione di oppresso - tramite la discussione su temi suscitati da un dibattito: la casa, la salute, il lavoro, la natura, i processi culturali, ecc. - e in seguito il processo di scrittura e di alfabetizzazione, che conduce a ciò che Freire chiama "liberazione". La liberazione non avviene mai in solitudine: è tutta la comunità del "circolo di cultura" che insieme si libera, prendendo coscienza dei meccanismi ingiusti della società.

Il rapporto tra educazione e politica nel pensiero di Freire pone efficacemente interrogativi sulla questione della gestione del potere. Educazione e politica sono due dimensioni dell'esperienza umana strettamente connesse nel suo pensiero così come nella vita del pedagogista brasiliano. Nella proposta pedagogica di Freire, esse sono saldate dal processo di coscientizzazione, poiché solo attraverso lo sviluppo di una coscienza critica e dialogica l'individuo può intraprendere azioni significative sul piano socio-politico.

Freire sottolinea così i rischi di un'educazione "neutrale", che non si schieri, che non prenda posizione, di cui vanno riconosciute le inadeguatezze e responsabilità storiche proprio nell'aver contribuito a sostenere regimi politici oppressivi: va ribadita allora la funzione sociale dell'educazione, che consiste essenzialmente nel produrre politica e società nuove.

Per evitare la riproduzione e il mantenimento dell'ideologia dominante, l'azione educativa, non essendo neutrale, impone di schierarsi: l'educatore aderisce, accetta, convive o con gli interessi dei dominanti o con quelli dei dominati, i cui diritti sono negati. Non è possibile invocare la neutralità di fronte al rapporto oppressore-oppresso, poiché inevitabilmente si favorirebbe il potere e, con esso, l'oppressore. Ogni educazione o è al servizio dell'oppressione oppure, se vuole evitare questo, deve necessariamente impegnarsi in un processo di liberazione; la stessa idea di libertà, inoltre, acquista il suo pieno significato solo quando si identifica con un impegno concreto degli uomini per la loro liberazione.

Si deve poi considerare il fatto che il progetto di alfabetizzazione di Freire sortiva conseguenze politiche in un paese come il Brasile, dove, negli anni Sessanta, a fronte di un basso numero di elettori (circa 15 milioni su una 
popolazione, con un età maggiore di 18 anni, di 34,5 milioni di persone $\left.{ }^{2}\right)$, una volta alfabetizzata la popolazione più povera sarebbe stata in grado di votare.

Gli interrogativi con il quale oggi Freire costringe a misurarsi sono essenzialmente due: quando educhiamo, come gestiamo il potere in nostro possesso? E inoltre, la nostra azione educativa contribuisce al mantenimento dell'ideologia dominante o propone il cambiamento nel mondo di strutture e meccanismi iniqui?

Le transizioni socio-culturali del nostro tempo possono essere comprese e affrontate con le chiavi interpretative e di intervento proposte da Freire.

La riflessione pedagogica ha affrontato in questi anni le problematiche relative all'incontro tra le diverse culture considerandola una tematica basilare. L'impegno in prima persona di educatore impone al pedagogista brasiliano di confrontarsi apertamente con la cultura altra, senza cedere ad alcuna tentazione etnocentrica ed evitando qualsiasi invasione culturale palese o cammuffata.

Il programma pedagogico di Freire è senza dubbio un progetto "interculturale", anzitutto nel suo tentativo di avviare un processo dinamico di crescita e di cambiamento nell'incontro con soggetti di diverse culture.

Il pedagogista brasiliano agisce nel rispetto dell'individualità del mondo di chi incontra, indipendentemente dal suo grado di alfabetizzazione: egli non cerca di trasmettere le conoscenze del mondo occidentale, ma di favorire nelle popolazioni del Sud del mondo lo sviluppo di un proprio senso critico.

Il carattere di interculturalità del progetto del pedagogista brasiliano si esprime poi nel suo impegno morale per ristabilire una posizione di reale parità tra le persone di differenti culture, in Brasile così come nelle altre parti del Sud del mondo.

Infatti, il dibattito pedagogico sull'interculturalità ha più volte sottolineato che nell'incontro tra persone che fanno riferimento a diverse culture uno dei due soggetti si trova in realtà in una situazione di subalternità, perché povero nel caso del Brasile, o soggetto a meccanismi iniqui nel Sud del mondo o, potremmo aggiungere, migrante o povero nel caso di un paese nel Nord del mondo.

Un progetto interculturale deve quindi essere legato anche ad un impegno morale per ristabilire equità tra i due soggetti della relazione, e l'impegno di Freire, attraverso l'unità dialettica tra teoria e prassi, riflessione e azione, è proprio diretto in questo senso, ossia a permettere agli individui il passaggio ad una nuova coscienza critica della propria situazione e al pieno esercizio dei propri diritti sociali e civili. 
Lo stesso metodo di alfabetizzazione di Paulo Freire apporta un altro contributo importante al dibattito pedagogico contemporaneo sull'interculturalità: in particolare, l'attenzione posta dal pedagogista brasiliano all'educazione permanente e le linee teoriche del suo metodo di alfabetizzazione forniscono principi e suggerimenti utilizzabili anche nei processi educativi degli stranieri adulti nel nostro paese, ad esempio nell'apprendimento dell' italiano come lingua seconda o nella formazione professionale.

La pedagogia di Freire resta quindi oggi un valido strumento rivolto a tutti gli "oppressi" del mondo, che necessitano di una prassi di liberazione dalla subalternità.

Il contributo di Freire è connesso, dunque, anche con le tematiche dell'educazione interculturale in quanto il suo approccio pedagogico può definirsi "decostruttivo": esso tende, appunto, a decostruire miti, pregiudizi, schemi mentali - sulla superiorità dei ricchi sui poveri, dei bianchi sui neri, dei leader politici sulle masse - che si sono diffusi in profondità in molti Paesi del Sud del mondo colonizzati dall'Occidente. Inoltre, è molto importante il concetto di "cultura" come sforzo creatore continuo dell'uomo, che mai si staticizza in un blocco monolitico ed è in costante mutamento e movimento. "L'invasione culturale è la penetrazione degli invasori nel contesto culturale degli invasi, senza rispetto verso le potenzialità dell'essere, che essa condiziona, quando essi impongono la loro visione del mondo e frenano la creatività, inibendo l'espansione degli invasi'" (FREIRE, 2002, p. 149). Per contro,

[...] nella sintesi culturale, che è l'opposto dell'invasione culturale, gli «attori» non arrivano al popolo come invasori, mentre nell'invasione culturale gli attori entrano dal loro mondo in quello degli invasi, portandovi un contenuto tematico per l'azione ricavato dai loro criteri di valore e dalla loro ideologia. Nella sintesi culturale gli attori, anche se arrivano da un «altro mondo», arrivano per conoscerlo col popolo e non per «insegnare», o trasmettere, o consegnare qualcosa al popolo (FREIRE, 2002, p.180).

Tutto ciò è affermato da Freire nella convinzione che

[...] ogni azione culturale è sempre una forma sistematica e deliberata di azione che incide sulla struttura sociale, ora nel senso di mantenerla com'è, ora nel senso di trasformarla... L'azione culturale, o è al servizio 
della dominazione (cosciente o incosciente da parte dei suoi agenti) o è al servizio della liberazione degli uomini (FREIRE, 2002, p.179).

L'impegno pedagogico, morale e politico di Paulo Freire è stato indubbiamente "profetico": la lettura delle sue opere, forse spesso dimenticate, ci esorta ancora oggi a pensare ed attuare una pedagogia degli oppressi.

Per le ragioni sopra esposte e non solo la Società Italiana di Pedagogia (SIPED) ha deciso di organizzare un convegno in suo onore tenutosi presso l'Università dell'Aquila nei giorni 24 e il 25 giugno 2021. Il Convegno dal titolo "Pedagogia e politica, in occasione dei 100 anni dalla nascita di Paulo Freire" ha ospitato gli interventi di alcuni dei maggiori studiosi a livello europeo (Peter Mayo e Marco Catarci), nonché delle giovani e giovanissime leve della pedagogia italiana.

Un convegno su Freire, ma anche intorno a Freire, per esplorare, approfondire, rilanciare la grande lezione dello studioso brasiliano, in cui il rapporto tra pedagogia e politica si fa inevitabile, intrinseco a qualsiasi scelta, progetto, azione che in educazione si porti avanti. In Freire questo rapporto è effettivamente molto stretto, in una visione dell'educazione carica di tensioni utopiche e trasformative, in cui si costruisce un'idea di mondo che veda come progetto, politico per l'appunto, la liberazione dai vincoli dell'oppressione, la coscientizzazione dei più deboli e degli esclusi, il pacifico e liberatore uso della parola come modo di prendere coscienza, reclamare e ottenere giustizia, senza creare nuovi vincoli di oppressione. Gli Atti del Convegno sono in corso di pubblicazione e, tuttavia, l'intero Convegno è disponibile in versione integrale sul sito della SIPED ${ }^{3}$.

\section{RIFERIMENTI}

CATARCI, Marco. La pedagogia della liberazione di Paulo Freire. Educazione, intercultura e cambiamento sociale. Milano: Franco Angeli, 2016.

FREIRE, Paulo. L'educazione come pratica della libertà. Milano: Mondadori, 1977.

FREIRE, Paulo. Pedagogia in cammino. Milano: Mondadori, 1979.

3 Disponibile in: https://www.siped.it. 
FREIRE, Paulo. La pedagogia degli oppressi., Torino: EGA, 2002.

FREIRE, Paulo. Pedagogia dell'autonomia. Torino: EGA, 2004.

TAGLIAVIA, Alfredo. L'eredità di Paulo Freire. Vita, pensiero, attualità pedagogica dell'educatore del mondo. Bologna: EMI, 2011.

Testo ricevuto il 14/10/2021.

Testo approvato il 28/10/2021. 